\title{
Calcipotriol Plus Betamethasone Dipropionate Aerosol Foam is Effective, Independent of Body Mass Index and the Extent and Severity of Psoriasis
}

Linda Stein Gold · John Villumsen · Monika Rosen

Received: August 23, 2016/Published online: October 6, 2016

(C) The Author(s) 2016. This article is published with open access at Springerlink.com

\begin{abstract}
Introduction: Good treatment adherence is important in the effective management of psoriasis and is related to both the frequency of applications and the amount of product used versus the recommended dose. The efficacy and safety of fixed combination calcipotriol $50 \mu \mathrm{g} / \mathrm{g}$ (Cal) and betamethasone $0.5 \mathrm{mg} / \mathrm{g}$ as dipropionate (BD) in the treatment of psoriasis is well established; an aerosol foam formulation has been developed to enhance adherence. This subanalysis from the Phase III PSO-FAST study evaluates the amount of Cal/BD foam used during treatment and the association between the extent and severity of baseline disease.
\end{abstract}

Methods: Patients ( $\geq 18$ years) with mild-to-severe body psoriasis were randomized 3:1 to once-daily Cal/BD foam or vehicle. The amount of Cal/BD foam and vehicle used over

Enhanced content To view enhanced content for this article go to http://www.medengine.com/Redeem/ D9E6F06027246DA3.

L. Stein Gold $(\square)$

Henry Ford Health System, Detroit, MI, USA

e-mail: 1stein1@hfhs.org

J. Villumsen · M. Rosen

LEO Pharma A/S, Ballerup, Denmark the 4-week study period was evaluated according to three baseline disease assessments: extent of body surface area (BSA) affected by psoriasis, physician's global assessment of disease severity (PGA) and modified psoriasis area and severity index (mPASI). Treatment success and mPASI75 rates were assessed according to body mass index (BMI) and body weight.

Results: 323 patients were randomized to $\mathrm{Cal} /$ BD foam and 103 to vehicle. At week 4, the mean total amount of Cal/BD foam used was $120.8 \mathrm{~g} \quad(n=293)$, which was similar to the amount of vehicle used (128.9 g; $n=98)$. The total amount of $\mathrm{Cal} / \mathrm{BD}$ foam used at week 4 was greater with increasing BSA and increasing severity of baseline PGA and mPASI. Throughout the study, $93.1 \%$ of patients in the Cal/BD foam group and $99.0 \%$ of patients in the vehicle group missed $\leq 10 \%$ of treatment applications. Treatment success and mPASI75 rates were generally similar when stratified according to BMI and body weight.

Conclusions: This subanalysis demonstrates that Cal/BD aerosol foam is used appropriately and is effective for the treatment of psoriasis, independent of BMI and the extent or severity of disease. 
Clinical trials number: NCT01866163.

Funding: LEO Pharma A/S.

Keywords: Calcipotriol; Betamethasone dipropionate; Aerosol foam; Psoriasis; Body mass index; Body surface area; Severity

\section{INTRODUCTION}

Therapeutic guidelines for psoriasis recommend the topical use of corticosteroids and vitamin $\mathrm{D}_{3}$ analogs as first-line treatment, either as separate products (used together) or as a fixed combination treatment [1]. Treatment adherence has been recognized as an important issue in the management of chronic inflammatory skin diseases such as psoriasis [2]. Although many patients can manage their psoriasis with topical therapy alone $[1,3,4]$, adherence remains a significant issue. Adherence is related to both the frequency of applications and the amount of product used compared with the recommended dose; a systematic literature review found that the frequency of topical treatment application in randomized controlled trials ranged from $55 \%$ to $100 \%$, while patients applied between $35 \%$ and $72 \%$ of the recommended dose [2]. A number of reasons for poor adherence to topical therapy were identified, including a lack of efficacy, excessive time applying the medication, and poor cosmetic characteristics [2]. Adherence was shown to be greater for once-daily treatment as compared with twice daily [2]. Studies have also shown that the topical vehicle used can impact on adherence $[5,6]$; patients generally prefer a vehicle that is simple and fast to apply, quickly absorbed and not greasy [7].

The efficacy and safety of the fixed combination of calcipotriol $50 \mu \mathrm{g} / \mathrm{g}$ (Cal) and betamethasone $0.5 \mathrm{mg} / \mathrm{g}$ as dipropionate (BD) has been confirmed in long-term trials [8-11]. The ointment and gel formulations of this fixed combination are established first-line treatments for psoriasis [12]. An aerosol foam formulation of the fixed $\mathrm{Cal} / \mathrm{BD}$ combination has been developed to enhance adherence and increase the therapeutic options available to patients. Previous Cal/BD aerosol foam studies have shown greater in vitro skin penetration compared with other formulations [13], and a significantly greater anti-psoriatic effect over 4 weeks of treatment than $\mathrm{Cal} / \mathrm{BD}$ ointment $[14,15]$, gel [16], and individual active ingredients [17], with a comparable tolerability profile [15-17]. In a large, double-blind, Phase III, 4-week study in patients with psoriasis vulgaris (PSO-FAST [NCT01866163]), treatment with $\mathrm{Cal} / \mathrm{BD}$ aerosol foam led to significantly greater treatment success rates $(53.3 \%$ vs. $4.8 \%$; $P<0.001)$ and lower mean modified psoriasis area and severity index (mPASI; excluding head; 2.0 vs $5.5 ; P<0.001)$ scores compared with aerosol foam vehicle [18].

This subanalysis from the PSO-FAST study evaluates the amount of Cal/BD aerosol foam used during treatment and the association between the extent and severity of baseline disease, in patients with psoriasis.

\section{METHODS}

\section{Patients and Study Design}

Patients eligible for inclusion in the PSO-FAST study were $\geq 18$ years of age with a clinical diagnosis of mild-to-severe body psoriasis. Patients had between $2 \%$ and $30 \%$ of their body surface area (BSA; i.e., trunk and limbs) affected by the disease, with an mPASI score of $\geq 2$. Full inclusion and exclusion criteria have been reported in Leonardi et al. [18]. 
PSO-FAST (Cal/BD aerosol foam in PSOriasis vulgaris, a Four-week, vehicle-controlled, efficacy And Safety Trial) was a Phase III, multicenter, double-blind study. Patients were randomized (3:1) to $\mathrm{Cal} / \mathrm{BD}$ aerosol foam or aerosol foam vehicle once daily for up to 4 weeks.

\section{Assessments}

The total amount of Cal/BD foam used by each patient during the 4-week study was determined by calculating the difference between the weight of the cans dispensed and the weight of the returned cans, multiplied by a correction factor of 0.41 to account for the propellant gases. Adherence was assessed by calculating the percentage of missed treatment applications ( $\leq 10 \%,>10-\leq 20 \%,>20-\leq 30 \%,>30-\leq 40 \%$, $>40-\leq 50 \%$ and $>50 \%$ ), as follows: the number of applications of Cal/BD foam missed for a particular visit interval was divided by the total number of days for the interval, multiplied by 100.

Treatment success was defined as the proportion of patients who were clear or almost clear of disease with at least a two-step improvement at week 4, according to the physician's global assessment of disease severity (PGA). The rate of treatment success was the primary endpoint of the study and the data have been reported in Leonardi et al. [18].

\section{Analysis}

The total amount of Cal/BD foam used during the study was evaluated according to three baseline disease assessments: the extent to which a patient's BSA was affected by psoriasis vulgaris; PGA; and mPASI. The proportion of patients achieving treatment success was stratified according to body weight and body mass index (BMI).

\section{Compliance with Ethics Guidelines}

The institutional review boards of all investigational sites approved the protocol. This study was conducted according to Good Clinical Practice guidelines and the principles of the Declaration of Helsinki 1964, as revised in 2013. All patients provided written informed consent prior to enrollment. The ClinicalTrials.gov identifier is NCT01866163.

\section{RESULTS}

In total, 323 patients were randomized to Cal/ BD foam and 103 to vehicle; 313 (96.9\%) and 99 (96.1\%) patients, respectively, completed the study. Of the 14 patients who withdrew, eight were lost to follow-up.

The overall mean ( \pm standard deviation) total amount of Cal/BD aerosol foam used after 4 weeks was $120.8 \pm 85.7 \mathrm{~g} \quad(n=293 / 323)$, which was similar to the mean total amount of aerosol foam vehicle used $(128.9 \pm 92.9 \mathrm{~g}$; $n=98 / 103)$. The mean amount of drug used per week was also similar between treatment groups: Cal/BD aerosol foam $29.8 \mathrm{~g} /$ week, vehicle $32.1 \mathrm{~g} /$ week. The mean amount of Cal/ BD aerosol foam used was consistent from baseline to week 1 (29.9 g/week), from week 1 to week 2 ( $28.5 \mathrm{~g} /$ week), and from week 2 to week 4 (30.2 g/week).

The total amount of Cal/BD aerosol foam used at week 4 was greater with increasing BSA and increasing severity of baseline PGA and mPASI (Fig. $1 \mathrm{a}-\mathrm{c}$ ). Most patients enrolled in the PSO-FAST study had $\leq 10 \%$ of their BSA involved at baseline $(n=227)$, and used on average between 88.3 and $117.6 \mathrm{~g}$ of $\mathrm{Cal} / \mathrm{BD}$ 

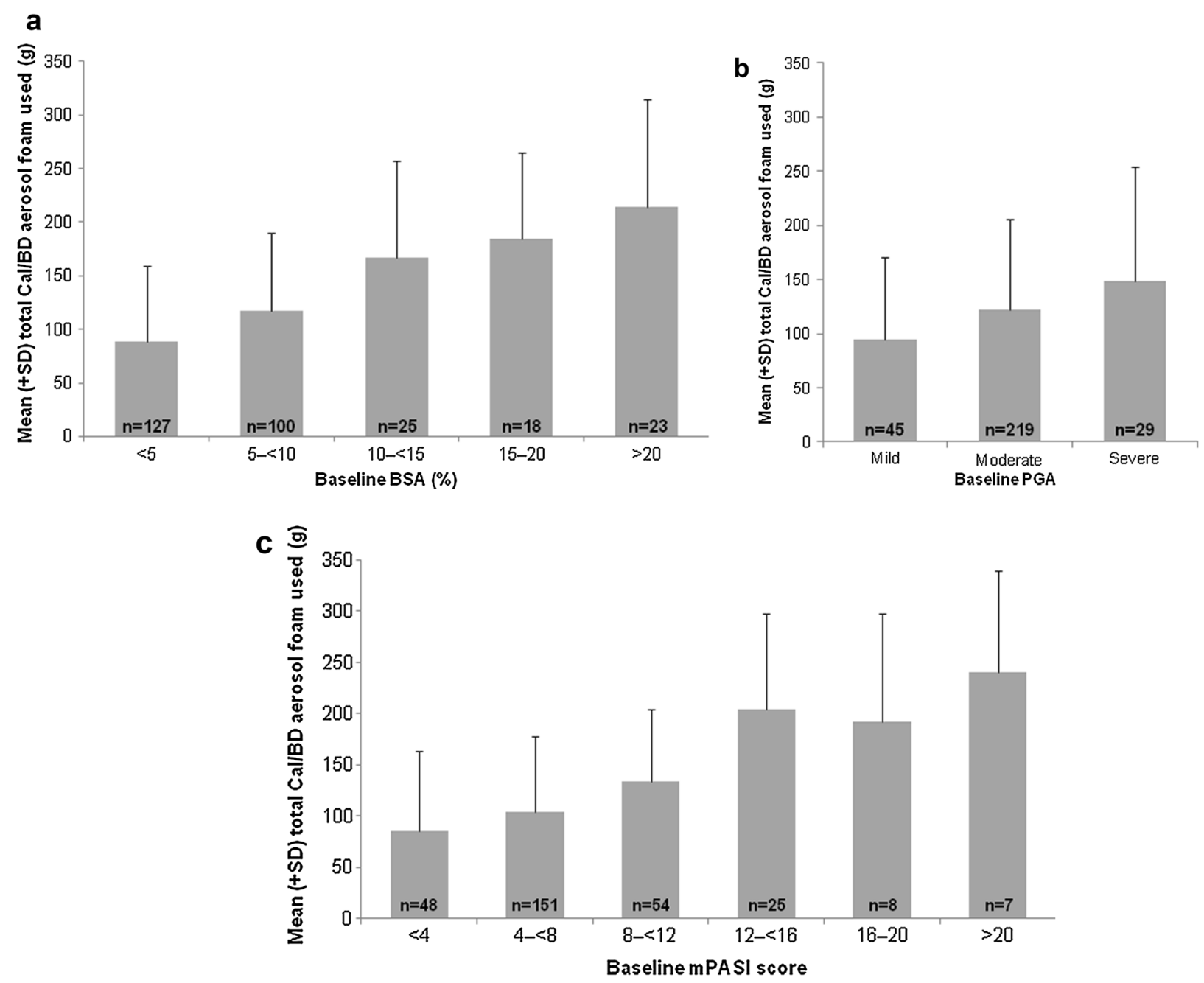

Fig. 1 Mean total amount of $\mathrm{Cal} / \mathrm{BD}$ aerosol foam used at week 4 by baseline a BSA; b PGA; and $\mathbf{c}$ mPASI. $B D$ betamethasone $0.5 \mathrm{mg} / \mathrm{g}$ as dipropionate, $B S A$ body surface area, Cal calcipotriol $50 \mu \mathrm{g} / \mathrm{g}, m P A S I$ modified psoriasis area and severity index, $P G A$ physician's global assessment of disease severity, $S D$ standard deviation

\section{DISCUSSION}

In the Phase III PSO-FAST study, most patients had mild-to-moderate disease severity (BSA $<10 \%$ ) and, on average, used $88-118 \mathrm{~g}$ of $\mathrm{Cal} / \mathrm{BD}$ foam. The amount of $\mathrm{Cal} / \mathrm{BD}$ aerosol foam used was comparable to vehicle and the product was used consistently throughout the 4-week study, suggesting that treatment persistence with the foam was good. In addition, patients used an appropriate amount of $\mathrm{Cal} / \mathrm{BD}$ aerosol foam for the extent and 

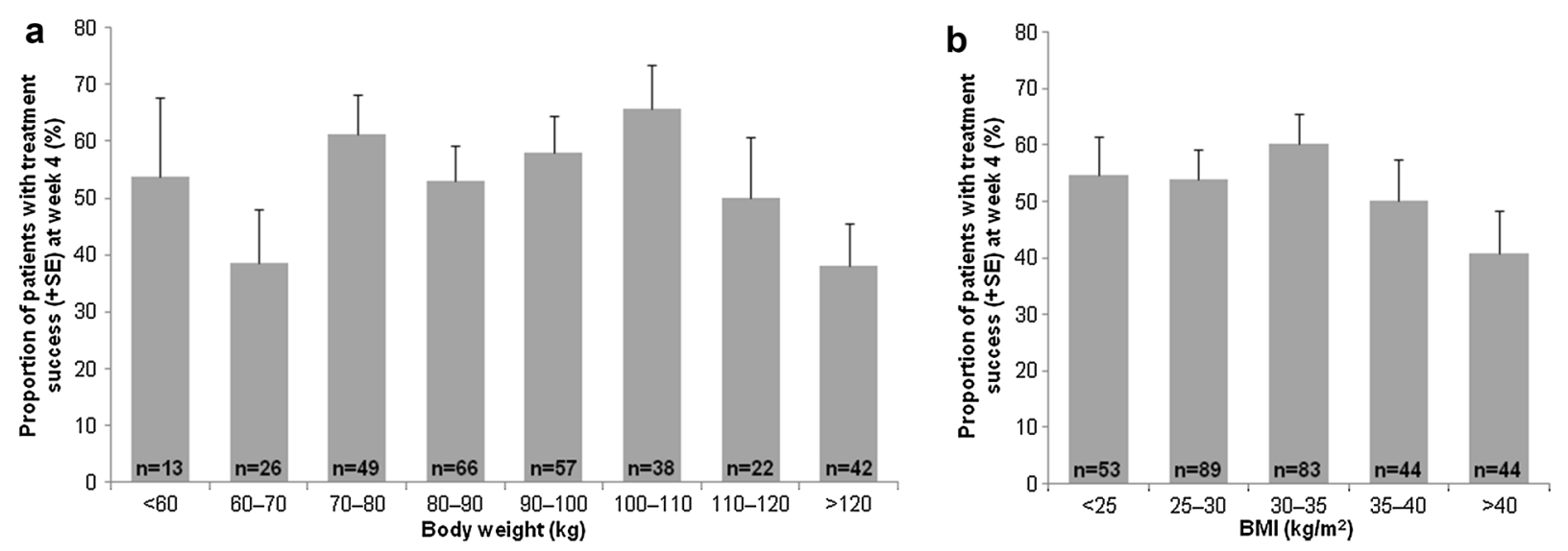

Fig. 2 Treatment success (defined as the proportion of patients who were clear or almost clear of disease with at least a two-step improvement at week 4, according to

severity of their psoriasis at baseline, since the mean total amount of Cal/BD aerosol foam used increased with increasing baseline BSA, PGA and mPASI.

The primary analysis demonstrated that significantly more patients using Cal/BD foam achieved treatment success versus vehicle and mean mPASI score was significantly lower for patients using Cal/BD foam than vehicle at week 4 [18]. This subanalysis shows that the effectiveness of $\mathrm{Cal} / \mathrm{BD}$ aerosol foam, based on treatment success rates, was not affected by body weight or BMI, so can be used in all patients irrespective of their size or extent of their BSA that is covered with plaques (as long as it is within the approved label). It has previously been suggested that BMI may be a prognostic factor for the response to treatment in psoriasis [19]; indeed, a number of studies have shown that some biologic therapies are less effective in patients with a BMI $\geq 25-30$ compared with a BMI <25 [19-23].

One limitation of this analysis is that the regular monitoring that occurred during this clinical study of relatively short duration may reinforce patient adherence compared with that
PGA) rates by a body weight and $\mathbf{b}$ BMI. BMI body mass index, $P G A$ physician's global assessment of disease severity, $S E$ standard error

observed in the real world. Outside of the clinical trial setting, some patients are unintentionally non-adherent with treatment (e.g., because they forget to use the medication as directed), whilst others are intentionally non-adherent (e.g., altering or stopping treatment because of perceived ineffectiveness or side effects) [24].

\section{CONCLUSION}

In conclusion, this subanalysis from the PSO-FAST study demonstrates that Cal/BD aerosol foam is used appropriately and is effective for the treatment of psoriasis, independent of BMI and the extent or severity of disease.

\section{ACKNOWLEDGMENTS}

Sponsorship and article processing charges for this study were funded by LEO Pharma A/S. Medical writing support was provided by Andrew Jones PhD, from Mudskipper Business Ltd, funded by LEO Pharma A/S. All authors had full access to all of the data in this study and 
take complete responsibility for the integrity of the data and accuracy of the data analysis. All named authors meet the International Committee of Medical Journal Editors (ICMJE) criteria for authorship for this manuscript, take responsibility for the integrity of the work as a whole, and have given final approval for the version to be published.

Disclosures. L. Stein Gold has been a consultant for LEO Pharma A/S and Pfizer, Novartis, Lilly, Galderma, Valeant, Taro, Activis, Allergan, has received honoraria from LEO Pharma A/S and Pfizer, Novartis, Lilly, Galderma, Valeant, Taro, Activis, Allergan, and has participated in speakers' bureaus for LEO Pharma A/S and Pfizer, Novartis, Lilly, Galderma, Valeant, Taro, Activis, Allergan. J. Villumsen is an employee of LEO Pharma A/S. M. Rosen is an employee of LEO Pharma $\mathrm{A} / \mathrm{S}$.

Compliance with Ethics Guidelines. The institutional review boards of all investigational sites approved the protocol. This study was conducted according to Good Clinical Practice guidelines and the principles of the Declaration of Helsinki 1964, as revised in 2013. All patients provided written informed consent prior to enrollment. The ClinicalTrials.gov identifier is NCT01866163.

Open Access. This article is distributed under the terms of the Creative Commons Attribution-NonCommercial 4.0 International License (http://creativecommons.org/licenses/ by-nc/4.0/), which permits any noncommercial use, distribution, and reproduction in any medium, provided you give appropriate credit to the original author(s) and the source, provide a link to the Creative Commons license, and indicate if changes were made.

\section{REFERENCES}

1. Menter A, Korman NJ, Elmets CA, Feldman SR, Gelfand JM, Gordon KB, et al. Guidelines of care for the management of psoriasis and psoriatic arthritis. Section 3. Guidelines of care for the management and treatment of psoriasis with topical therapies. J Am Acad Dermatol. 2009;60:643-59.

2. Devaux S, Castela A, Archier E, Gallini A, Joly P, Misery L, et al. Adherence to topical treatment in psoriasis: a systematic literature review. J Eur Acad Dermatol Venereol. 2012;26(Suppl 3):61-7.

3. Lebwohl M, Ting PT, Koo JY. Psoriasis treatment: traditional therapy. Ann Rheum Dis. 2005;64(Suppl 2):ii83-6.

4. Nast A, Boehncke WH, Mrowietz U, Ockenfels HM, Philipp S, Reich K, et al. German S3-guidelines on the treatment of psoriasis vulgaris (short version). Arch Dermatol Res. 2012;304:87-113.

5. Puig L, Carrascosa JM, Belinchon I, Fernandez-Redondo $\mathrm{V}, \quad$ Carretero G, Ruiz-Carrascosa JC, et al. Adherence and patient satisfaction with topical treatment in psoriasis, and the use, and organoleptic properties of such treatments: a Delphi study with an expert panel and members of the Psoriasis Group of the Spanish Academy of Dermatology and Venereology. Actas Dermosifiliogr. 2013;104:488-96.

6. Tan X, Feldman SR, Chang J, Balkrishnan R. Topical drug delivery systems in dermatology: a review of patient adherence issues. Expert Opin Drug Deliv. 2012;9:1263-71.

7. Eastman WJ, Malahias S, Delconte J, DiBenedetti D. Assessing attributes of topical vehicles for the treatment of acne, atopic dermatitis, and plaque psoriasis. Cutis. 2014;94:46-53.

8. Kragballe K, Austad J, Barnes L, Bibby A, de la Brassinne M, Cambazard F, et al. Efficacy results of a 52-week, randomised, double-blind, safety study of a calcipotriol/betamethasone dipropionate two-compound product (Daivobet ${ }^{\circledR} /$ Dovobet $^{\circledR} /$ Taclone $\left.{ }^{\circledR}\right)$ in the treatment of psoriasis vulgaris. Dermatology. 2006;213:319-26.

9. Kragballe K, Austad J, Barnes L, Bibby A, de la Brassinne M, Cambazard F, et al. A 52-week randomized safety study of a calcipotriol/betamethasone dipropionate two-compound product (Dovobet ${ }^{\circledR} /$ Daivobet $^{\circledR} /$ Taclonex $^{\circledR}$ ) in the treatment of psoriasis vulgaris. Br J Dermatol. 2006;154:1155-60.

10. Lambert J, Hol CW, Vink J. Real-life effectiveness of once-daily calcipotriol and betamethasone 
dipropionate gel vs. ointment formulations in psoriasis vulgaris: final analysis of the 52-week PRO-long study. J Eur Acad Dermatol Venereol. 2015;29:2349-55.

11. Luger TA, Cambazard F, Larsen FG, Bourcier M, Gupta G, Clonier F, et al. A study of the safety and efficacy of calcipotriol and betamethasone dipropionate scalp formulation in the long-term management of scalp psoriasis. Dermatology. 2008;217:321-8.

12. Laws PM, Young HS. Topical treatment of psoriasis. Expert Opin Pharmacother. 2010;11:1999-2009.

13. Hollesen Basse L, Olesen M, Lacour JP, Queille-Roussel C. Enhanced in vitro skin penetration and antipsoriatic effect of fixed combination calcipotriol plus betamethasone dipropionate in an innovative foam vehicle. J Invest Dermatol. 2014;134:S33:abst 192.

14. Queille-Roussel C, Olesen M, Villumsen J, Lacour JP. Efficacy of an innovative aerosol foam formulation of fixed combination calcipotriol plus betamethasone dipropionate in patients with psoriasis vulgaris. Clin Drug Investig. 2015;35:239-45.

15. Koo J, Tyring S, Werschler WP, Bruce S, Olesen M, Villumsen J, et al. Superior efficacy of calcipotriene and betamethasone dipropionate aerosol foam versus ointment in patients with psoriasis vulgaris-a randomized phase II study. J Dermatol Treat. 2016;27:120-7.

16. Paul C, Stein Gold L, Cambazard F, Kalb RE, Lowson D, Bang B, et al. Calcipotriol plus betamethasone dipropionate aerosol foam provides superior efficacy versus gel in patients with psoriasis vulgaris: randomized, controlled PSO-ABLE study. J Eur Acad Dermatol Venereol. 2016. doi:10.1111/ jdv.13859.

17. Lebwohl $\mathrm{M}$, Tyring $\mathrm{S}$, Bukhalo $\mathrm{M}$, Alonso-Llamazares J, Olesen M, Lowson D, et al. Fixed combination aerosol foam calcipotriene
$0.005 \%$ (Cal) plus betamethasone dipropionate $0.064 \%(\mathrm{BD})$ is more efficacious than Cal or BD aerosol foam alone for psoriasis vulgaris: a randomized, double-blind, multicenter, three-arm, phase II study. J Clin Aesthet Dermatol. 2016;9:34-41.

18. Leonardi C, Bagel J, Yamauchi P, Pariser D, Xu Z, Olesen $M$, et al. Efficacy and safety of calcipotriene plus betamethasone dipropionate aerosol foam in patients with psoriasis vulgaris-a randomized phase III study (PSO-FAST). J Drugs Dermatol. 2015;14:1468-77.

19. Naldi L, Addis A, Chimenti S, Giannetti A, Picardo $\mathrm{M}$, Tomino $\mathrm{C}$, et al. Impact of body mass index and obesity on clinical response to systemic treatment for psoriasis. Evidence from the Psocare project. Dermatology. 2008;217:365-73.

20. Cassano N, Galluccio A, De Simone C, Loconsole F, Massimino SD, Plumari A, et al. Influence of body mass index, comorbidities and prior systemic therapies on the response of psoriasis to adalimumab: an exploratory analysis from the APHRODITE data. J Biol Regul Homeost Agents. 2008;22:233-7.

21. Umezawa Y, Saeki H, Nakagawa H. Some clinical factors affecting quality of the response to ustekinumab for psoriasis. J Dermatol. 2014;41:690-6.

22. Yanaba K, Umezawa Y, Ito T, Hayashi M, Kikuchi S, Fukuchi O, et al. Impact of obesity on the efficacy of ustekinumab in Japanese patients with psoriasis: a retrospective cohort study of 111 patients. Arch Dermatol Res. 2014;306:921-5.

23. Puig L. Obesity and psoriasis: body weight and body mass index influence the response to biological treatment. J Eur Acad Dermatol Venereol. 2011;25:1007-11.

24. Richards HL, Fortune DG, Griffiths CE. Adherence to treatment in patients with psoriasis. J Eur Acad Dermatol Venereol. 2006;20:370-9. 